\title{
Análisis del significado institucional sobre los enfoques probabilísticos en el nivel medio superior, un caso de estudio
}

\author{
Ilseth Johana Leyva Zazueta \\ Manuel Alfredo Urrea Bernal \\ Eleazar Silvestre Castro
}

\begin{abstract}
Resumen: En este trabajo se realiza un análisis de corte cualitativo del plan de estudio para la Educación Media Superior y un libro de texto vigente para bachillerato tecnológico mexicano, en torno a los enfoques probabilísticos. Partimos de las herramientas teóricas del Enfoque Ontosemiótico para determinar el tratamiento actual otorgado a estas nociones. Los resultados muestran ciertas discrepancias entre ambas herramientas de análisis en tanto al uso y concentración de los conceptos identificados para estos, se precisa de modificaciones importantes en la matemática escolar a fin de lograr mejores resultados de aprendizaje y desarrollo adecuado de razonamiento probabilístico.
\end{abstract}

Palabras clave: enfoques de probabilidad. objetos matemáticos. bachillerato.

Ilseth Johana Leyva Zazueta Licenciatura em 2021 pela Universidad de Sonora.

Hermosillo, Sonora, México.

iD https://orcid.org/0000-0002-4940-1724

$\triangle$ ilsethleyva@hotmail.com

Manuel Alfredo Urrea Bernal Mestrado em 2002 pela Universidad de

Sonora, campus Hermosillo. Professor da Universidad de Sonora (UNISON), Hermosillo, Sonora, México.

Dhttp://orcid.org/0000-0002-0496-6385

$\triangle$ manuel.urrea@unison.mx

Eleazar Silvestre Castro Doutorando em 2019 pela Centro de Investigaciones y Estudios Avanzados del Instituto Politécnico Nacional

(CINVESTAV-IPN), campus Zacatenco.

Professor da Universidad de Sonora (UNISON), Hermosillo, Sonora, México.

http://orcid.org/0000-0002-9472-483X

$\triangle$ eleazar.silvestre@unison.mx

Recebido em 30/06/2021 Aceito em 08/09/2021 Publicado em 30/10/2021

\section{Analysis of the institutional meaning of probabilistic approaches in high school, a case study}

Abstract: In this work, a qualitative analysis of the study plan for high school and a current textbook for Mexican technological high school is carried out, around probabilistic approaches. We start from the theoretical tools of the Ontosemiotic Approach to determine the current treatment given to these notions. The results show certain discrepancies between both analysis tools in terms of the use and concentration of the concepts identified for them, it is necessary to make important modifications in school mathematics to achieve better learning results and adequate development of probabilistic reasoning.

Keywords: probabilistic approaches. primary objects. high school.

\section{Análise do significado institucional das abordagens probabilísticas no nível secundário superior, um estudo de caso}

Resumo: Neste trabalho é realizada uma análise qualitativa do plano de estudos do Ensino Médio e de um livro didático atual para o ensino médio mexicano de tecnologia, em torno de abordagens probabilísticas. Partimos das ferramentas teóricas da Abordagem Ontosemiótica para determinar o tratamento atual dado a essas noções. Os resultados mostram certas discrepâncias entre as duas ferramentas de análise no que diz respeito ao uso e concentração dos conceitos identificados, pois estas, importantes modificações são necessárias na matemática escolar, a fim de alcançar melhores resultados de aprendizagem e desenvolvimento adequado do raciocínio probabilístico.

Palavras-chave: significados de probabilidade. objetos primários. ensino médio. 


\section{Introducción}

A lo largo de la historia de la humanidad, los requerimientos formativos han ido evolucionando y con esto se han modificado cada vez más las directrices curriculares para todos los niveles educativos. En particular, observamos en cada propuesta cada vez más contenido y nociones probabilísticas para niveles previos al superior (LEYVA, 2021). Lo anterior atendiendo las necesidades actuales de la población, pues es de gran importancia brindar a los individuos los medios que les permitan el desarrollo de capacidades para la toma de decisiones adecuadas frente a diversas situaciones de la vida diaria. Es así como el estudio de la probabilidad se vuelve una herramienta importante para el desarrollo del razonamiento probabilístico de la sociedad; al respecto, Batanero (2006) señala la importancia su estudio, por ser la probabilidad una disciplina matemática base para otros campos científicos y por su constante aparición en la vida diaria.

Entenderemos al razonamiento probabilístico como el desarrollo de las siguientes cualidades en los estudiantes: "identificar eventos aleatorios en la naturaleza, tecnología y sociedad, analizar las condiciones y derivar los supuestos para un modelado apropiado, construir modelos matemáticos, explorar diversos escenarios y resultados de estos modelos, aplicar métodos y procedimientos matemáticos de probabilidad y estadística" (BATANERO et al., 2016, p. 9). Es decir, el razonamiento probabilístico corresponderá a la forma en la que los estudiantes vinculan y modifican sus conocimientos de referencia acerca de la probabilidad para constituir los conceptos relacionados con los enfoques de probabilidad mediante la identificación, análisis y modelación de situaciones estocásticas.

Investigadores coinciden en que el razonamiento probabilístico se desarrolla de forma incipiente y en ocasiones inadecuada por la utilización de materiales didácticos, como es el caso de los libros de texto escolares, que reproducen significados parciales o erróneos respecto a los normativos (por ejemplo, que otorgan mayor énfasis al tratamiento de un enfoque probabilístico sobre otro, presentan definiciones confusas u omiten supuestos importantes para el cumplimento de propiedades, entre otras) (GÓMEZ; CONTRERAS y BATANERO, 2015). Por esta razón, en este trabajo nos interesamos por el significado establecido sobre los enfoques de probabilidad, tanto en un libro de texto como en un programa curricular del nivel Medio Superior de Educación en México. Para ello, presentamos una síntesis de un análisis de corte cualitativo realizado en torno a los enfoques de probabilidad del programa para la Educación Media Superior (EMS) y un análisis de un texto escolar para el bachillerato tecnológico actualmente en uso para la asignatura de Probabilidad y Estadística, así como un contraste entre ambas unidades de análisis. 


\section{Antecedentes}

De las principales dificultades identificadas para un desarrollo adecuado del razonamiento probabilístico, se destaca la presencia de un énfasis desproporcionado concedido a la probabilidad clásica, concentrando su estudio a técnicas de repetición y combinatorias para el cálculo de probabilidades cuantitativas (GODINO; BATANERO y CAÑIZARES,1996). De acuerdo con Valdez (2016), la enseñanza de la probabilidad se favorece del enfoque Laplaciano por encima de los restantes y, aunque el enfoque frecuencial si se encuentra presente en la matemática escolar, no hay una clara relación de este con el enfoque frecuencial. En nuestra consideración, la fuerte presencia del sesgo de equiprobabilidad (GREEN, 1983; CAÑIZARES, 1997; GÓMEZ, CONTRERAS y BATANERO, 2015; BEGUÉ et al., 2017; LEYVA, 2021) respalda, en parte, este énfasis sobre el enfoque clásico en la enseñanza de la probabilidad.

Esta inclinación hacia la probabilidad desde su enfoque teórico provoca que, por ejemplo, los individuos busquen reducir cada situación aleatoria a la aplicación de la regla de Laplace. Por su parte, Inzunza (2017), afirma que el énfasis hacia la probabilidad clásica conduce a restar importancia a las demás perspectivas y, menciona que los tres enfoques presentados en forma coordinada propiciarían una mejora en las intuiciones y razonamiento probabilístico de los educandos.

Gómez et al. (2015) analizan el significado de probabilidad de dos series de textos escolares para educación primaria y la comparan con las directrices curriculares correspondientes; sus resultados muestran que los textos desarrollan parcialmente cuatro enfoques probabilísticos: se resalta la presencia de mayoritaria del enfoque intuitivo, seguido del estudio de los enfoques clásico y frecuencial de forma más reducida, mientras que el enfoque subjetivo se aborda de forma tangencial. Las dos series analizadas presentan características muy diferentes, uno más centrado en temas estadísticos y otro aborda las nociones probabilísticas de manera más amplia, de igual forma, se destaca en ambos la ausencia del uso de tecnología para realizar simulaciones. En particular, una de las series se inclina hacia el estudio de la probabilidad clásica y omite el enfoque frecuencial lo que puede conducir a la presencia del sesgo de equiprobabilidad, mientras que la otra propone el estudio de la probabilidad frecuencial, pero no realiza suficientes reproducciones para los experimentos aleatorios propuestos, lo que puede conducir inducir o reforzar la heurística de la representatividad.

En la misma línea, Vázquez y Alsina (2015), proponen un modelo para el análisis de cómo se plantea la probabilidad en textos matemáticos de educación primaria. De igual forma, presentan el análisis de una colección de textos matemáticos chilenos, en los que se observa el predominio 
del enfoque intuitivo, seguido del estudio de los enfoques clásico y frecuencial en forma progresiva y finalmente el enfoque subjetivo en forma breve. Los autores identifican discrepancias entre lo establecido curricularmente y lo encontrado en los textos analizados, y determinan la necesidad de una reformulación del tratamiento probabilístico presente en los textos que aspire a un pensamiento probabilístico gradual mediante situaciones cercanas al estudiante que incluyan los enfoques de probabilidad establecidos por el programa de estudio.

Adicionalmente, un elemento que ha mostrado ser de gran ayuda para la enseñanza y aprendizaje de la probabilidad ha sido el uso de tecnología (INZUNZA, 2017). En particular, en el plan de estudio para el bachillerato tecnológico se hace hincapié en la importancia de uso de la tecnología tanto para el dominio de estas herramientas (competencias necesarias para la vida diaria y laboral), como para orientar el aprendizaje a partir de ellas, que desprende el término Tecnologías del Aprendizaje y del Conocimiento (TAC), estas promueven la enseñanza mediante metodologías de aplicación de herramientas tecnológicas, para conocer y explorar la gama de posibilidades didácticas que ofrece una herramienta (SEP, 2017). No obstante, el mismo Inzunza (2017) destaca que tales directrices curriculares se tocan de manera tangencial, pues no se articulan de manera precisa y adecuada con los procesos de simulación y experimentación que ofrecen distintos programas computacionales destinados a complementar y desarrollar significado acerca de múltiples conceptos probabilísticos (por ejemplo, Tinkerplots).

Estos elementos nos presentan una serie escenarios y problemáticas en el tratamiento actual de la matemática escolar asociada a los enfoques probabilísticos, de los cuales destacamos una inclinación hacia el estudio de la probabilidad clásica, que puede conducir al desarrollo de sesgos y heurísticas, así como la disparidad existente en diversos análisis de la probabilidad entre los establecido en los planes de estudio y libros de texto. Lo que nos indica la necesidad de replantear la matemática escolar en torno a los enfoques de probabilidad y brindar un panorama general que logre apoyar de manera efectiva al desarrollo del razonamiento probabilístico de los estudiantes y los faculte hacia la toma efectiva de decisiones presentes en la vida diaria.

\section{Referentes teóricos}

\subsection{Significados de probabilidad}

La probabilidad admite diversos enfoques que se complementan entre sí y que, dadas sus características, desencadenan dificultades para su enseñanza (BATANERO, 2005). En relación con esto, muchos autores remarcan que los diferentes enfoques probabilísticos se deben estudiar 
de forma articulada, evitando limitar su enseñanza hacia un enfoque en particular (BATANERO; HENRY y PARZYSZ, 2005); es decir, se deben buscar presentar estas nociones a los estudiantes de una forma gradual, mediante un estudio dosificado durante su paso por el sistema educativo (Batanero, 2005). De acuerdo con esta autora, los enfoques probabilísticos se resumen en:

Enfoque intuitivo: Se refiere a las ideas intuitivas de azar y e incertidumbre que manifiesta un individuo haya sido o no instruido en probabilidad.

Enfoque clásico: "fracción cuyo numerador es el número de casos favorables y cuyo denominador es el número de todos los casos posibles" (LAPLACE, 1985/1914, p.28), conciliable con situaciones aleatorias que posean espacios muestrales finitos y eventos equiprobables.

Enfoque frecuencial: "valor hipotético hacia el cual tiende la frecuencia relativa de un suceso al estabilizarse, asumiendo la repetibilidad del ensayo" (GÓMEZ et al., 2015), se admite la existencia de dicho límite y la frecuencia relativa representa una aproximación al valor teórico de probabilidad.

Enfoque subjetivo: Se asume que la probabilidad de un evento aleatorio será determinada por evaluaciones propias de la mente de un sujeto individual y no características del mundo en el que vivimos, se establece bajo el supuesto de que las personas tendrán valores de probabilidad individuales derivados de un patrón de preferencia implícito entre decisiones (BOROVNIK y KAPADIA, 2014).

Enfoque axiomático: Aunque ampliamente utilizada, las definiciones anteriores representan ciertos inconvenientes desde puntos de vista matemáticos, por lo que, para la teoría de probabilidad moderna, se desarrolla el enfoque matemático - axiomático, donde el concepto de probabilidad como tal, no se define. Se considera la probabilidad como un tipo especial de medida que se construye axiomáticamente partiendo de la teoría de conjuntos y de la medida, este enfoque ha sido de gran aceptación y sus aplicaciones se extienden en diversos campos de estudio.

En particular, nos interesamos por determinar la forma en la que se presentan estas nociones dentro del sistema didáctico y cómo se relaciona con la forma en la que los estudiantes vinculan y modifican sus nociones en torno a los enfoques probabilísticos, así como la manera en la que se abren paso hacia el desarrollo de un razonamiento probabilístico que los faculte hacia la toma de decisiones oportunas frente a diversas circunstancias. 


\subsection{Enfoque Ontosemiótico de la Enseñanza y la Instrucción Matemáticos}

En este trabajo se consideran elementos teóricos del Enfoque Ontosemiótico del Conocimiento y la Instrucción Matemáticos (EOS) (GODINO y BATANERO, 1994; GODINO, BATANERO y FONT, 2008; GODINO et al., 2021) para realizar los análisis alrededor de los enfoques de probabilidad. En un principio, se establece a las prácticas matemáticas como noción primitiva, definidas como "toda actuación o manifestación (lingüística o no) realizada por alguien para resolver problemas matemáticos, comunicar a otros la solución, validar la solución y generalizarla a otros contextos y problemas" (GODINO y BATANERO, 1994, p. 8), y que pueden ser realizadas por una persona o desarrollarse en el seno de una institución (un grupo de individuos que intervienen en un mismo tipo de situaciones problema) (GODINO et al., 2008).

También se adopta la noción de objeto matemático, considerado como toda entidad que intervenga en los sistemas de prácticas matemáticas y que permitan su individualización. La noción de práctica matemática da pie al nacimiento del concepto de significado de un objeto matemático, referente al sistema de prácticas discursivas y operativas, a fin de dar solución a determinado tipo o familia de problemas (GODINO; CONTRERAS y FONT, 2006). Por otro lado, se establecen los siguientes tipos de significados institucionales (GODINO et al., 2008, p. 5):

Implementado: sistema de prácticas efectivamente implementadas por el docente.

Evaluado: el subsistema de prácticas que utiliza el docente para evaluar los aprendizajes.

Pretendido: sistema de prácticas incluidas en la planificación del proceso de estudio.

Referencial: sistema de prácticas que se usa como referencia para elaborar el significado pretendido.

Para este proyecto se tomarán en cuenta los significados: pretendido a través del análisis a libros de texto y referencial por medio del análisis a planes y programas de estudio. Respecto a los significados personales, distinguimos los tipos (GODINO et al., 2008):

Global: globalidad del sistema de prácticas personales que logra manifestar potencialmente el sujeto respecto a un objeto matemático.

Declarado: prácticas efectivamente expresadas por el sujeto.

Logrado: prácticas expresadas por el sujeto que están alineadas al significado institucional de referencia. 
Así, el aprendizaje se dará "mediante la participación del sujeto en las comunidades de práctica, el acoplamiento progresivo de los significados personales a los institucionales y la apropiación de los significados institucionales por los estudiantes" (GODINO et al., 2008, p. 5). Se propone entonces, la siguiente tipología de objetos matemáticos primarios (ver Figura 2) (GODINO, et al., 2008):

Elementos lingüísticos: términos, expresiones, notaciones, gráficos (diversos registros).

Situaciones - problemas: ejercicios, aplicaciones extra o intramatemáticas.

Concepto - definición: entidades matemáticas que se pueden introducir a través de definiciones o descripciones.

Proposiciones: propiedades, atributos o enunciados que se utilizan para dar solución a una situación problema.

Procedimientos: algoritmos, operaciones, técnicas de cálculo, que los estudiantes despliegan para dar solución a las situaciones problema.

Argumentos: enunciados usados para la validación o explicación de proposiciones y procedimientos para resolver una situación problema determinada.

Ahora bien, los objetos matemáticos primarios se articulan para generar nuevas entidades, llamaremos configuración a "las redes de objetos intervinientes y emergentes de los sistemas de prácticas y las relaciones que se establecen entre los mismos" (GODINO, et al., 2008, p. 8).

\section{Elementos metodológicos}

Esta es una metodología de tipo cualitativa. A fin de presentar el análisis y confrontación de la forma actual en la que existen los diferentes enfoques de probabilidad en el bachillerato tecnológico mexicano, adoptamos la metodología propuesta en Vázquez y Alsina (2015), que retoman elementos del EOS para el análisis de objetos matemáticos primarios y sus significados presentes en textos matemáticos y directrices curriculares. En este sentido, retomamos las seis fases propuestas por los autores adaptadas para la EMS (llustración 1): buscamos identificar cómo se presentan estas nociones en la EMS, cómo se ponen en juego, qué significado se les otorga en ambos elementos de análisis, sus relaciones y las configuraciones que forman. 
llustración 1. Modelo de análisis de los objetos matemáticos primarios y sus significados en libros de texto.

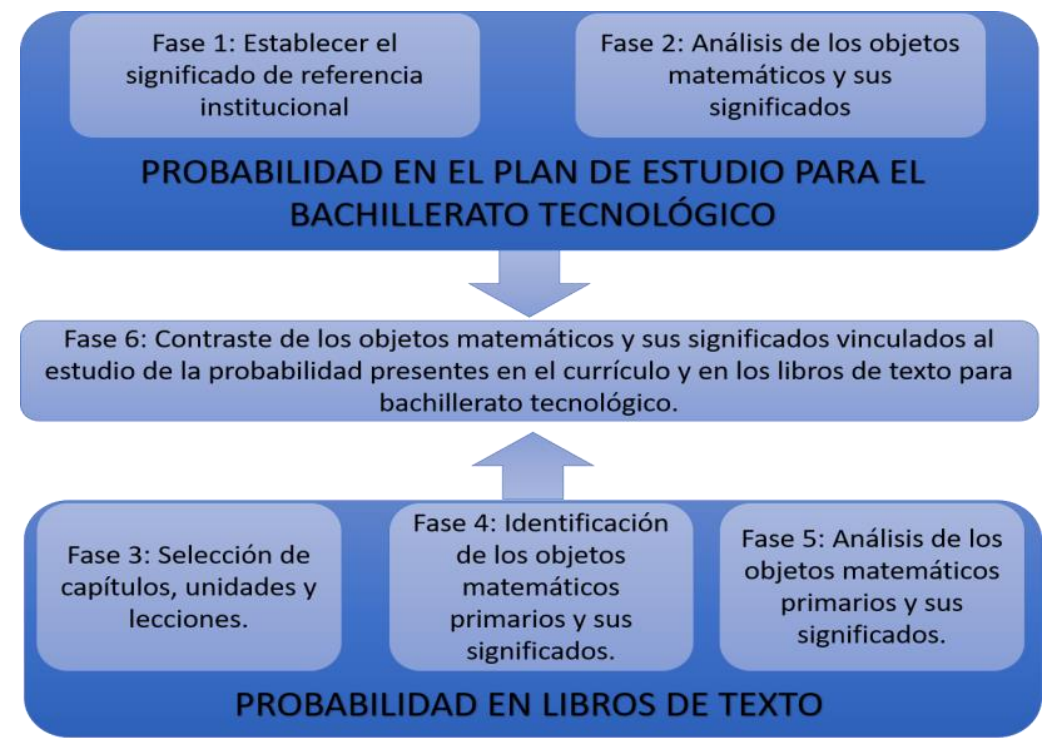

Fuente: Adaptación Vázquez y Alsina (2015).

En las fases 1 y 2, se busca establecer el significado de referencia institucional de los enfoques de probabilidad a partir de un análisis del plan de estudio de bachillerato tecnológico mexicano. Para ello tomamos como base al Programa de estudios del componente básico del marco curricular común de la Educación Media Superior (SEP, 2017), identificando y analizando los objetos matemáticos primarios y sus significados para los diferentes enfoques de probabilidad.

En las fases 3, 4 y 5 presentamos el análisis cualitativo del libro de texto Probabilidad y Estadística para bachilleratos tecnológicos (SALAZAR, 2018). El texto fue seleccionado por ser actualmente utilizado en la asignatura de Probabilidad y Estadística para el Centro Tecnológico de Estudios del Mar. El libro de texto fue facilitado por un docente de la asignatura y es especializado para bachilleratos tecnológicos, por lo que representa una fuente de análisis viable para apoyar la identificación del significado de referencia. Para su análisis, se seleccionan los capítulos que aborden el estudio de los enfoques de probabilidad, mismos que se exhiben en la siguiente tabla:

Tabla 1. Desglose de unidades del texto seleccionado que desarrollan los enfoques de probabilidad.

\begin{tabular}{|l|l|c|}
\hline \multicolumn{1}{|c|}{ Unidad } & \multicolumn{1}{|c|}{ Lección (es) } & Páginas \\
\hline Eje. Del manejo de & 1.1 Nociones y conceptos básicos de estadística y probabilidad & $8-16$ \\
\cline { 2 - 3 } $\begin{array}{l}\text { la información al } \\
\text { pensamiento } \\
\text { estocástico. }\end{array}$ & $\begin{array}{l}\text { 1.2 Enfoques de probabilidad ¿Qué significa cada enfoque de } \\
\text { probabilidad?, ¿qué significan las medidas de tendencia central?, } \\
\text { ¿primera parte }\end{array}$ & $17-26$ \\
\cline { 2 - 3 } & $\begin{array}{l}\text { ¿pué obtener estos valores? } \\
\text { determinación de probabilidades }\end{array}$ & $27-46$ \\
\cline { 2 - 3 } & ClERRE & $47-49$ \\
\hline
\end{tabular}

Fuente: Salazar (2018) 
En particular, seleccionamos la lección 1.2 como elemento de análisis, puesto que los ejercicios, problemas y ejemplos desarrollan los temas de interés.

En la fase 6, realizamos un contraste entre los resultados obtenidos a partir del análisis del plan de estudio y del texto escolar seleccionado, por medio de confrontar los resultados y determinar las coincidencias, discrepancias identificadas e implicaciones en torno al contenido matemático de interés.

\section{Resultados}

\subsection{Análisis de objetos y sus significados presentes en el plan de estudio}

Realizamos un análisis al programa de estudio para bachilleratos tecnológicos mexicanos alrededor de los enfoques de probabilidad para identificar el significado de referencia, del cual distinguimos las siguientes prácticas matemáticas asociadas al estudio de la probabilidad:

- Permitir que el estudiante construya las nociones y conceptos ligados a la probabilidad y estadística.

- Plantear problemas cercanos a la realidad del estudiante, que motiven hacia el aprendizaje de la probabilidad y la estadística y, que hagan explícito el valor de uso de los conocimientos asociados.

- Registro, interpretación y análisis de datos estadísticos en contextos de aleatoriedad para realizar estimaciones y adquirir competencias hacia la toma de decisiones.

- Adquisición de conocimientos en torno a la probabilidad y estadística para su aplicación dentro y fuera de contextos escolares.

- Explicitar los vínculos entre la probabilidad y la estadística.

- Vinculación de nociones de probabilidad y estadística con otras áreas propias de la matemática y otros campos de estudio.

- Planteamiento de problemas que evidencien la importancia del estudio de la probabilidad.

Seguido, presentamos los objetos matemáticos primarios (situaciones problema, elementos lingüísticos, conceptos - definición y proposiciones) identificados en los planes de estudio para la EMS en relación con los enfoques de probabilidad.

Situaciones problema: De acuerdo con lo establecido curricularmente, se plantea el abordar los enfoques de probabilidad que no incluyan el estudio de distribuciones de probabilidad, por ser conceptos reservados para niveles superiores de educación. De esta forma, en la Tabla 2 
presentamos las situaciones problema identificadas para los enfoques intuitivos, clásico, frecuencial y subjetivo de probabilidad y su correspondencia con cada uno de ellos:

Tabla 2. Situaciones problema presentes en el plan de estudios para bachillerato tecnológico

\begin{tabular}{|l|}
\hline \multicolumn{1}{|c|}{ Situaciones problema } \\
\hline SP1. Identificación de situaciones de azar, incertidumbre y aleatoriedad. \\
SP2. Determinación/identificación del espacio muestral de un experimento aleatorio basado en técnicas de \\
conteo (implícito). \\
SP3. Recolección, organización, clasificación y/o interpretación de datos para resolución de problemas \\
estadísticos, situaciones de azar, aleatoriedad e incertidumbre. \\
SP4. Estimación de la probabilidad de eventos a partir del análisis de datos. \\
SP5. Cálculo de probabilidad de ocurrencia de un evento aleatorio. \\
SP6. Análisis de problemas donde a partir de información nueva la probabilidad de un evento puede modificarse \\
para la aplicación de probabilidades condicionales. \\
SP7. Formulación e interpretación de problemas para hacer uso de los diferentes enfoques probabilísticos. \\
SP8. Investigación, presentación y exposición de nociones y conceptos estadísticos/probabilísticos. \\
SP9. Análisis de datos estadísticos / probabilísticos.
\end{tabular}

Fuente: Elaboración propia.

Por otro lado, es importante resaltar que el programa no menciona explícitamente el estudio de problemas relacionados con el enfoque intuitivo, por lo que no se incluyen situaciones problemas del tipo: Estimación de probabilidades de eventos aleatorios cualitativamente a partir de grados de creencia personal.

Por último, el estudio del enfoque subjetivo de probabilidad se establece implícitamente a partir de una aproximación al Teorema de Bayes. Por tanto, no incluimos situaciones del tipo: Estimación de probabilidades de eventos aleatorios a partir del grado de creencia personal con base en experimentación, conocimiento e información proporcionada por expertos.

Elementos lingüísticos: Se identifican los siguientes elementos lingüísticos asociados a los enfoques de probabilidad

Tabla 3. Elementos lingüísticos en plan de estudio de bachillerato tecnológico

\begin{tabular}{|l|}
\hline \multicolumn{1}{|c|}{ Elementos lingüísticos } \\
\hline Lenguaje verbal/natural \\
Lenguaje simbólico \\
Lenguaje gráfico \\
Lenguaje numérico \\
Lenguaje algebraico \\
Lenguaje figural \\
\hline
\end{tabular}

Fuente: Elaboración propia.

Conceptos - Definiciones: Respecto a los conceptos y definiciones presentes en el programa de estudio para la EMS referentes a los enfoques probabilísticos, identificamos la presencia de forma explícita y, de igual forma, podemos asociar otros conceptos de forma implícita. Por otra parte, también se identifica la omisión de conceptos que a nuestra consideración son 
importantes para el desarrollo de los conceptos probabilísticas para los enfoques intuitivo y subjetivo. La siguiente tabla muestra cada uno de estos conceptos - definiciones.

Tabla 4. Conceptos - definiciones en plan de estudio bachillerato tecnológico.

\begin{tabular}{|l|l|l|}
\hline \multicolumn{1}{|c|}{ Presentes de forma explícita } & \multicolumn{1}{c|}{ Implícitos } & \multicolumn{1}{c|}{ Ausentes } \\
\hline Probabilidad & Ensayo/reproducción del & Suerte, destino \\
Colección de datos & experimento. & Evento seguro, posible, \\
Azar & Espacio muestral & probable, imposible, \\
Fenómeno aleatorio - no aleatorio & Conjunto & predecible. \\
(determinista - no determinista) & Conjunto finito - infinito & Grado de creencia \\
Incertidumbre & Equiprobabilidad & personal \\
Aleatoriedad & Estimación & \\
Enfoques de probabilidad & Ley de los Grandes Números & \\
Técnicas de conteo & Juego justo / injusto & \\
Riesgo & Experimento con o sin reemplazo. & \\
Eventos & & \\
Independencia/dependencia de eventos & & \\
Experimento aleatorio & & \\
Frecuencia absoluta/relativa & & \\
\hline
\end{tabular}

Procedimientos: los procedimientos relacionados a los diferentes enfoques probabilísticos se identifican de forma explícita e implícita dentro del programa de estudio, de igual forma encontramos una omisión en procedimientos referentes a los enfoques intuitivo y subjetivo de probabilidad.

Tabla 5. Procedimientos en plan de estudio de bachillerato tecnológico.

\begin{tabular}{|c|c|c|}
\hline Explícitos & Implícitos & Omisiones \\
\hline $\begin{array}{l}\text { PRE1. Análisis de resultados de } \\
\text { experimentos aleatorios. } \\
\text { PRE2.Lectura, diseño y/o } \\
\text { interpretación de tablas (diversos } \\
\text { tipos). } \\
\text { PRE3. Aplicación de la Regla de } \\
\text { Laplace para el cálculo de } \\
\text { probabilidades cualitativas. } \\
\text { PRE4. Cálculo de frecuencias } \\
\text { relativas. } \\
\text { PRE5. Organización de resultados. } \\
\text { PRE6. Interpretación de resultados } \\
\text { y estimación de probabilidades. } \\
\text { PRE7. Aplicación de encuestas } \\
\text { para recopilar datos. }\end{array}$ & $\begin{array}{l}\text { PRI1. Identificación y presentación de } \\
\text { espacio muestral. } \\
\text { PRI2. Distinción de eventos } \\
\text { favorables - no favorables del } \\
\text { espacio muestral. } \\
\text { PRI3. Identificación de características } \\
\text { de un suceso aleatorio determinado } \\
\text { (cardinalidad del espacio muestral e } \\
\text { independencia / no independencia de } \\
\text { eventos). } \\
\text { PRI4. Reproducción de experimentos } \\
\text { conservando las condiciones } \\
\text { iniciales. } \\
\text { PRI5. Comparación de la estimación } \\
\text { de probabilidad obtenida por métodos } \\
\text { experimentales con la probabilidad } \\
\text { obtenida teóricamente. }\end{array}$ & $\begin{array}{l}\text { PRO1. Distinción entre los } \\
\text { tipos de situaciones } \\
\text { aleatorias. } \\
\text { PRO2. Reconocimiento de } \\
\text { escalas cualitativas de } \\
\text { probabilidad. } \\
\text { PRO3. Estimación cualitativa } \\
\text { de probabilidades asociadas } \\
\text { a sucesos aleatorios. } \\
\text { PRO4. Análisis de situaciones } \\
\text { donde la probabilidad recae } \\
\text { en información basada en } \\
\text { conocimientos previos, } \\
\text { grados de creencia personal. }\end{array}$ \\
\hline
\end{tabular}

Fuente: Elaboración propia. 


\subsection{Análisis de objetos y sus significados en libros de texto: "calcula la probabilidad relativa"}

En el libro de texto Probabilidad y Estadística para bachilleratos tecnológicos (SALAZAR, 2018), se hace mención del enfoque intuitivo y se aborda el estudio del enfoque frecuencial, clásico y axiomático de manera explícita y el subjetivo mediante el estudio de la probabilidad condicional. Aunque hemos realizado un análisis de la unidad didáctica completa, en lo siguiente, mostramos los objetos matemáticos identificados para la actividad Calcula la probabilidad relativa (véase Anexo 1), que pertenece a la lección 1.2 (p. 20-24), por ser de las actividades que consideramos más representativas de entre las que plantea el texto.

Antes de presentar la identificación de los objetos matemáticos primarios que corresponden a esta actividad, es importante mencionar los elementos previos que se introducen en el texto (lo que da inicio a la etapa de institucionalización). En esta parte, se enuncian los diferentes enfoques que se aceptan para la probabilidad, aunque no se incluye al enfoque subjetivo, aun cuando el plan de estudio menciona su estudio relacionado con el Teorema de Bayes. Por otro lado, el enfoque intuitivo se establece como "probabilidad empírica" sin declarar que también es conocida como frecuencial, de frecuencia relativa, experimental, entre otras; consideramos que esto puede conducir a confusiones en los estudiantes respecto a la identificación y diferenciación de cada enfoque probabilístico. Acto seguido, el texto presenta una definición de probabilidad empírica (enfoque intuitivo), clásica y frecuencial y dos ejemplos de aplicación de estos.

Observamos que en la definición de probabilidad relativa se introduce el término experimento aleatorio, considerándolo como "aquel que puede ser reproducido un número indefinido de ocasiones", pero no especifica el rasgo no determinista que lo distingue. Finalmente, se presenta la actividad "Calcula la probabilidad relativa", misma que presenta los siguientes objetos matemáticos primarios:

Tabla 6. Identificación de objetos matemáticos primarios: Ejercicio 6.

\begin{tabular}{l} 
Situación - Problema \\
\hline Para esta actividad se identifican dos tipos de situaciones problema: \\
SP3. Recolección, organización, clasificación y/o interpretación de datos para resolución de problemas \\
estadísticos, situaciones de azar, aleatoriedad e incertidumbre. \\
Al recolectar resultados de ensayos del experimento aleatorio (ya sea de lanzamiento de monedas o \\
del giro de pirinola). \\
SP4. Estimación y análisis de la probabilidad de eventos a partir del análisis de datos.
\end{tabular}




\begin{tabular}{|c|c|}
\hline \multicolumn{2}{|c|}{$\begin{array}{l}\text { Al realizar estimaciones de probabilidad de los resultados experimentales y dar respuesta a los } \\
\text { cuestionamientos dados a partir de las estimaciones. }\end{array}$} \\
\hline \multicolumn{2}{|c|}{ Lenguaje matemático } \\
\hline \multicolumn{2}{|c|}{ Verbal, tabular, simbólico y numérico } \\
\hline \multicolumn{2}{|c|}{ Conceptos - Definiciones } \\
\hline $\begin{array}{l}\text { Probabilidad Previos } \\
\text { Experimento aleatorio } \\
\text { Azar / aleatoriedad } \\
\text { Espacio muestral } \\
\text { Evento } \\
\text { Ensayo }\end{array}$ & $\begin{array}{l}\text { Emergentes } \\
\text { Frecuencia (absoluta/relativa) } \\
\text { Límite } \\
\text { LGN } \\
\text { Estimación } \\
\text { Probabilidad frecuencial } \\
\text { Probabilidad clásica }\end{array}$ \\
\hline \multicolumn{2}{|c|}{ Proposiciones } \\
\hline \multicolumn{2}{|c|}{$\begin{array}{c}\text { Si tenemos una sucesión de variables aleatorias independientes e idénticamente distribuidas con varianza finita, } \\
\text { entonces el promedio de las } n \text { primeras observaciones (variables aleatorias) se acerca a la media teórica } \\
\text { cuando el número n de repeticiones tiende hacia infinito. }\end{array}$} \\
\hline Procedimientos & Argumentos \\
\hline $\begin{array}{l}\text { PRI. Identificación y presentación del espacio } \\
\text { muestral } \\
\text { Reproducción de un experimento aleatorio una } \\
\text { cantidad finita de veces. }\end{array}$ & $\begin{array}{l}\text { La frecuencia relativa de los resultados de un cierto } \\
\text { experimento aleatorio, tienden a estabilizarse en cierto } \\
\text { número, cuando el experimento se realiza muchas } \\
\text { veces, lo que nos permite estimar la probabilidad por } \\
\text { medios experimentales. }\end{array}$ \\
\hline Organización de resultados en forma tabular. & \\
\hline $\begin{array}{l}\text { PRE6. Interpretación de resultados y estimación de } \\
\text { probabilidades. }\end{array}$ & \\
\hline
\end{tabular}

Fuente: Elaboración propia.

Respecto a la estructura, previo a la actividad se hace la institucionalización de los conceptos y definiciones. La actividad en sí se divide en dos secciones: una etapa de apertura limitada a presentar propósitos, conocimientos previos y materiales y una etapa de desarrollo donde se desglosan dos experimentos aleatorios (lanzamiento de dados y giro de pirinola), no se incluye una sección de cierre para la actividad.

Esta actividad se trabaja de forma grupal y aborda los conceptos referentes al enfoque frecuencial. Se identifican dos tipos de situaciones problema: SP3. Recolección, organización, clasificación y/o interpretación de datos para resolución de problemas de aleatoriedad y SP4. Estimación y análisis de la probabilidad de eventos a partir del análisis de datos. Estas situaciones problemas se pueden entender en términos de dos momentos para la resolución de la actividad; en un inicio se pide la reproducción de ensayos y organización de resultados en tablas de frecuencia (SP3) para dos situaciones de aleatoriedad, posteriormente se hacen estimaciones de probabilidad a partir de los resultados y análisis de la concordancia de los resultados obtenidos conformados en equipo y después integrando los resultados de todos los equipos del grupo (SP4).

Respecto a los conceptos que moviliza la actividad, reconocemos como previos: probabilidad, Azar / Aleatoriedad, experimento aleatorio, evento, espacio muestral, ensayo y como 
conceptos que se movilizan durante la actividad: frecuencia, límite, LGN, estimación, probabilidad frecuencial. Las situaciones problema darán cabida a las proposiciones relacionadas con el enfoque frecuencial de probabilidad (justificados por las argumentaciones), y, a su vez, estas darán lugar al uso de procedimientos específicos.

Identificamos que, en general, se plantean dos experimentos aleatorios, el primero acerca del lanzamiento de un dado de seis caras y el segundo el giro de una pirinola de seis lados (es decir, con características similares que guardan la condición de equiprobabilidad); así mismo, para cada uno de los experimentos se realizan los mismos cuestionamientos. Consideramos que se podría apoyar más al aprendizaje de los estudiantes el promover otro tipo de situaciones más allá de la condición de equiprobabilidad, que permitan enriquecer las experiencias de los estudiantes, en el sentido de que pongan en juego prácticas diferentes y complementarias, por ejemplo, vía las condiciones donde haya una variante y el espacio muestral no sea equiprobable, para conducir al estudiante a identificar lo distintivo y útil de cada enfoque probabilístico.

Por otro lado, consideramos que el número de reproducciones de los experimentos aleatorios resultan insuficientes para lograr estimaciones adecuadas de probabilidad en cada una de las situaciones aleatorias; al ser una cantidad relativamente pequeña de reproducciones, los resultados (frecuencias absolutas o relativas) pueden variar considerablemente respecto al valor teórico. Se propone entonces el uso de softwares de simulación que complementen a la experimentación física y que permitan la reproducción del experimento una mayor cantidad de veces, de esta forma el estudiante podrá observar la convergencia de los resultados al modelo probabilístico esperado.

\subsection{Contraste de objetos matemáticos primarios, sus significados y configuraciones presentes en el plan de estudio y libro de texto.}

Finalmente, presentamos un análisis centrado en los objetos matemáticos primarios presentes en el plan de estudio y libro de texto seleccionado referente a los enfoques probabilísticos. Notamos que, aunque el plan de estudio plantea el abordar aquellos enfoques que no requieran el estudio de distribuciones de probabilidad, los enfoques intuitivo y subjetivo no se hacen presentes en gran medida en ambas unidades de análisis. En particular, el libro de texto limita el enfoque intuitivo a un listado de ejemplos y el enfoque subjetivo se estudia de forma implícita en lecciones posteriores. Los enfoques clásico y frecuencial se estudian de manera resumida, la actividad seleccionada para análisis es la única que aborda estos enfoques de forma 
explícita, para proseguir con la presentación del enfoque axiomático (donde identificamos el estudio de actividades relacionadas con el enfoque clásico).

En general, consideramos que el libro de texto seleccionado atiende las orientaciones curriculares establecidas para el bachillerato tecnológico de manera parcial. Destacamos la falta de construcción de los conceptos por parte del estudiante; el estudio de la probabilidad se presenta enfatizado a juegos de azar y no tanto en situaciones de la realidad del estudiante y que evidencien la importancia de la probabilidad; y la ausencia del uso de las nociones probabilísticas para promover el desarrollo de competencias necesarias para la toma de decisiones. Adicionalmente, se observa en esta unidad de análisis la falta de uso de herramientas de tecnología digital, contrastando con lo establecido curricularmente.

\section{Conclusiones}

A partir de los análisis que hemos realizado, consideramos que el tratamiento que se le da a los enfoques de probabilidad dentro del libro de texto seleccionado no se encuentra en total correspondencia con las directrices curriculares establecidas. Esto se evidencia en la forma de institucionalización de conceptos, presentados previo a las actividades, lo que conduce a que los estudiantes las admitan como tal, sin la posibilidad de construirlas por ellos mismos. También notamos una alta concentración de situaciones de juegos de azar para el estudio de la probabilidad. En particular, para la probabilidad clásica y frecuencial, el texto se limita a una sola actividad (seleccionada para análisis) que aborda dos problemas de juegos de azar, esto resalta la inclinación existente hacia el estudio de juegos de azar. De igual forma, observamos ciertas inconsistencias respecto al significado normativo en las definiciones planteadas por el libro de texto, como el caso de la definición de probabilidad intuitiva (presentada como empírica) y la definición de experimento aleatorio.

En relación con la tecnología, el plan de estudio establece el uso de herramientas digitales tanto para su dominio como para orientación del aprendizaje, lo que no se hace presente en lo observado en el libro de texto analizado. Referente a los enfoques probabilísticos, observamos el uso de tecnología limitado a la presentación o búsqueda de contenido, pero no para la orientación del aprendizaje, lo cual coincide con los resultados de las investigaciones de Gómez et al. (2015). En nuestra perspectiva, un uso más apropiado de estos recursos sería, por ejemplo, recurrir a simuladores de experimentos aleatorios para la vinculación de los enfoques clásico y frecuencial 
de la probabilidad, al permitir al estudiante identificar la estabilización de frecuencias relativas aumentando el número de reproducciones del experimento.

Por otro lado, en el libro de texto seleccionado no se identifica la presencia del énfasis señalado hacia el estudio de la probabilidad desde su enfoque teórico, pues se observa una clara intención de vincular la probabilidad clásica y frecuencial dentro de la actividad analizada. Aunque los dos enfoques se encuentran presentes en el texto escolar, determinamos que no se realiza una clara diferenciación entre estos, si bien la actividad "Calcula la probabilidad relativa" busca hacer esta conexión mediante experimentación, a partir del análisis realizado se evidencia la falta de desarrollo de estas nociones que resalten los atributos que caracterizan y diferencian a los enfoques clásico y frecuencial. Finalmente, los resultados obtenidos a partir de este análisis coinciden con los resultados reportados en los estudios de Gómez et al. (2015), pues la actividad analizada se limita a pocas reproducciones de los experimentos aleatorios, que pueden tener una gran variación de resultados y conducir a la presencia de sesgos y heurísticas.

Por lo anterior, se evidencia la necesidad de replantear la forma de presentación de los conceptos probabilísticos para que el alumno logre enriquecer sus prácticas respecto a los enfoques de probabilidad, que lleven a mejorar los significados logrados por los estudiantes, así como desarrollar competencias necesarias tanto para niveles de estudio superior, como situaciones dentro y fuera de contextos matemáticos e incorporando diversos tipos de herramientas didácticas para el apoyo de los aprendizajes.

\section{Referencias}

BATANERO, Carmen. Significados de la probabilidad en la educación secundaria. Revista Latinoamericana de Investigación en Matemática Educativa, 8(3), 247-264, 2005.

BATANERO, Carmen. Razonamiento probabilístico en la vida cotidiana: Un desafío educativo. Investigación en el aula de matemáticas. Estadística y azar, Sociedad de Educación Matemática Thales. 2006.

BATANERO, Carmen, HENRY, Michel y PARZYSZ, Bernard. The nature of chance and probability. En Graham A Jones (Ed.). Exploring probability in school: Challenges for teaching and learning. 15-37. New York: Springer. 2005.

BATANERO, Carmen; CHERNOFF, Egan J.; ENGEL, Joachim; LEE, Hollylynne S.; SÁNCHEZ, Ernesto. Research on Teaching and Learning Probability. ICME-13 Topicar Surveys Cham: Springer. 2016.

BEGUÉ, Nuria; BATANERO, Carmen; GEA, Maria Magdalena; BELTRAN-PELLICER, pablo. Comprensión del enfoque frecuencial de la probabilidad por estudiantes de Educación Secundaria 
Obligatoria. En J.M. Muñoz-Escolano, A. Arnal-Bailera, P. Beltrán-Pellicer, M.L. Callejo, \& J. Investigación en Educación Matemática XXI. p. 137-146, 2017.

BOROVCNIK, Manfred; KAPADIA, Ramesh. A historical and philosophical perspective on probability. E. J Chernoff, y B. Sriraman, (Eds.), Probabilistic thinking: presenting plural perspectives. p. 7-34. Springer Netherlands. 2014.

CAÑIZARES, M. Jesús. Influencia del razonamiento proporcional y combinatorio y de creencias subjetivas en las intuiciones probabilísticas primarias. 1997. Tesis Doctoral. Universidad de Granada.

GODINO, Juan D; BATANERO. Carmen. Significado institucional y personal de los objetos matemáticos. Recherches en Didactique des Mathématiques, 14 (3): 325-355. 1994.

GODINO, Juan D.; BATANERO, Carmen; Cañizares, M. Jesús. Azar y probabilidad. Fundamentos didácticos y propuestas curriculares. Madrid: Síntesis.1987.

GODINO, Juan D; BATANERO, Carmen; FONT, Vicenç. Un enfoque ontosemiótico del conocimiento y la instrucción Matemática, versión ampliada y revisada del artículo de GODINO, Juan D; BATANERO, Carmen; FONT, Vicenç. "The onto semiotic approach to research in mathematics education". The International Journal on Mathematics Education, v. 39, (1-2): $p$. 127-135. 2008.

GODINO. Juan C; CONTRERAS, Ángel; FONT, Vicenç. Análisis de procesos de instrucción basado en el enfoque ontológico-semiótico de la cognición matemática. Recherches en Didactiques des Mathematiques. 26(1), p 39-88. 2006.

GODINO, Juan D.; BATANERO, Carmen; BURGOS, María; GEA, María M. Uma perspectiva ontosemiótica dos problemas e métodos de pesquisa em educação matemática. Revemop, v. 3 , p. e202107, 21 jun. 2021. Disponível em: https://doi.org/10.33532/revemop.e202107

GÓMEZ, Emilse; BATANERO, Carmen; CONTRERAS, José Miguel. Conocimiento matemático de futuros profesores para la enseñanza de la probabilidad desde el enfoque frecuencial. Boletim de Educação Matemática, 28(48), 209-229. 2014.

GÓMEZ, Emilse; BATANERO, Carmen; CONTRERAS, José Miguel. Significados de la probabilidad en libros de texto para Educación Primaria en Andalucía. En C. Fernández, M. Molina y N. Planas (eds.), Investigación en Educación Matemática XIX. p. 73-87. Alicante: SEIEM. 2015.

GREEN, David, R. A Survey of probabilistic concepts in 3000 pupils aged 11-16 years. En D. R. Grey et al. (Eds.), Proceedings of the First International Conference on Teaching Statistics. v.2, p. 766 - 783. University of Sheffield. 1983.

INSUNZA, Santiago. Conexiones entre Probabilidad Teórica y Probabilidad Frecuencial en un Ambiente de Modelación Computacional. Avances de investigación en educación matemática, 11, 69-86. 2017.

LAPLACE, Pierre Simon. Ensayo filosófico sobre las probabilidades. Madrid, España: Alianza Editorial (Trabajo original publicado en 1814). 1985. 
LEYVA, Ilseth. Análisis didáctico referido a la probabilidad frecuencial. 2021. Tesis de Licenciatura, Universidad de Sonora. México.

SALAZAR, Guerrero, Ludwing. Probabilidad y Estadística para Bachilleratos Tecnológicos. Serie DEGTI. México: Patria Educación. 2018.

SEP. Secretaría de Educación Pública.Programa de estudios del componente básico del marco curricular común de la Educación Media Superior. Campo disciplinar matemáticas. Bachillerato tecnológico. Probabilidad y Estadística. 2017.

VALDEZ, Julio César. Las grandes ideas de probabilidad en el razonamiento informal de estudiantes de bachillerato. 2016. Tesis Doctoral. Centro de Investigación y de Estudios Avanzados del Instituto Politécnico Nacional. México.

VÁZQUEZ, Claudia. y ALSINA, Ángel. Un modelo para el análisis de objetos matemáticos en libros de texto chilenos: situaciones problemáticas, lenguaje y conceptos sobre probabilidad. Profesorado. Revista de Currículum y Formación de Profesorado, 19(2), 441-462. 2015.

\section{Anexo 1.}

Actividad Calcula la probabilidad relativa (Salazar, 2018, p. 20 - 24)

Apertura de la actividad

Deduce y aprende

Calcula la probabilidad relativa

Propósito: Determinar la probabilidad relativa de la cara de un dado.

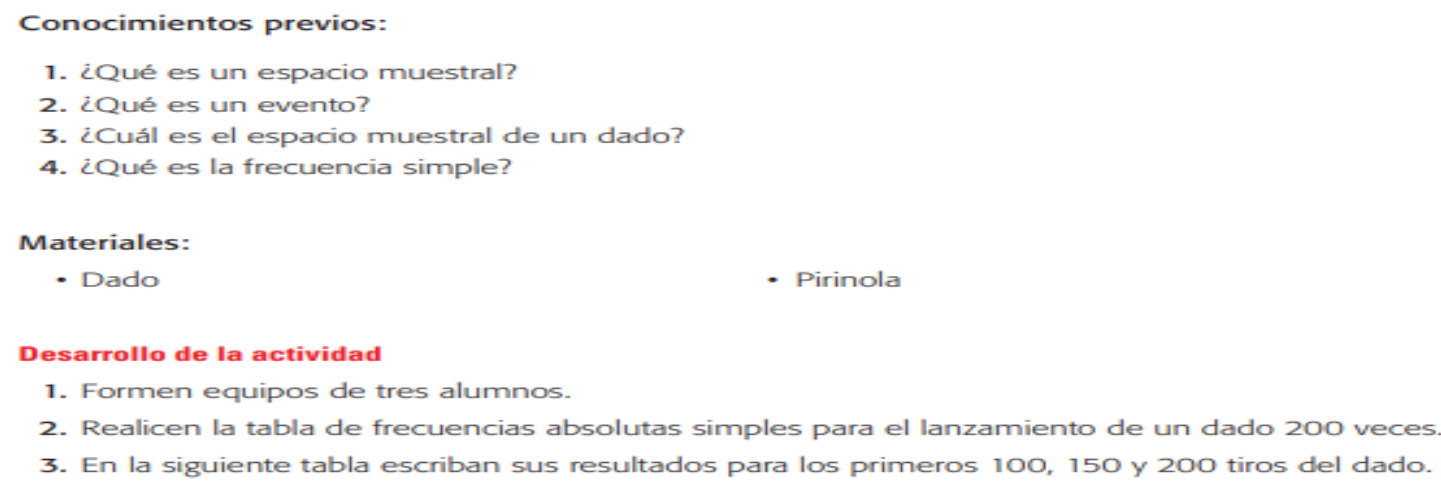

Tabla 1.4
\begin{tabular}{|c|c|c|c|} 
Cara del dado & Para 100 & Para 150 & Para 200 \\
\hline 1 & & & \\
\hline 2 & & & \\
\hline 3 & & & \\
\hline 4 & & & \\
\hline 5 & & & \\
\hline 6 & & & \\
\hline
\end{tabular}


4. ¿Cuál es el espacio muestral del experimento?

5. La probabilidad de un evento es igual al cociente de las frecuencias absolutas simples entre el total de las frecuencias. Este cociente recibe el nombre de

Calculen las siguientes probabilidades:
$P(1)=$
$P(2)=$
$P(3)=$
$P(4)=$
$P(5)=$
$P(6)=$

La primera de ellas se lee: "La probabilidad de obtener un uno al tirar un dado es igual a

6. ¿Todas las probabilidades son iguales?

7. Cuando $N$ (el número de tiradas crece) y la probabilidad tienden a estabilizarse hacia un número en particular, ¿cuál es?

8. Comparen este número con el de sus compañeros. ¿Se parecen?

9. Anoten en la siguiente tabla los resultados que obtuvieron sus compañeros para el caso de 200 lanzamientos.

\begin{tabular}{|c|c|c|c|c|c|c|c|}
\hline & & $P(1)$ & $P(2)$ & $P(3)$ & $P(4)$ & $P(5)$ & $P(6)$ \\
\hline \multirow{11}{*}{ 음 } & A & & & & & & \\
\hline & B & & & & & & \\
\hline & C & & & & & & \\
\hline & D & & & & & & \\
\hline & $\mathrm{E}$ & & & & & & \\
\hline & $\mathrm{F}$ & & & & & & \\
\hline & G & & & & & & \\
\hline & $\mathrm{H}$ & & & & & & \\
\hline & I & & & & & & \\
\hline & J & & & & & & \\
\hline & $\mathrm{K}$ & & & & & & \\
\hline \multicolumn{8}{|c|}{ Suma } \\
\hline \multicolumn{2}{|c|}{ Promedio } & & & & & & \\
\hline
\end{tabular}

10. Sumen las probabilidades de cada columna y obtengan su promedio.

11. ¿Todas las probabilidades son iguales?

12. Cuando $N$ (el número de tiradas), crece, ula probabilidad tiende a estabilizarse hacia un número en particular? ¿Cuál?

13. ¿La probabilidad $P(i)$ tiende a estabilizarse hacia un número en particular? ¿Cuál? ¿A cuál?

14. Si tiran el dado y cae cinco, ¿̇la siguiente tirada se verá afectada por este resultado? ¿Por qué? 
15. Realicen la tabla de frecuencias absolutas simples para el giro de una pirinola 100 veces.

16. En la siguiente tabla escriban los resultados para los primeros 75,100 y 125 giros de la pirinola.

Tabla 1.6 Frecuencias absolutas simples

\begin{tabular}{|c|c|c|c|} 
Cara de la pirinola & Para 75 & Para 100 & Para 125 \\
\hline Todos ponen & & & \\
\hline Toma uno & & & \\
\hline Toma dos & & & \\
\hline Toma todo & & & \\
\hline Pon uno & & & \\
\hline Pon dos & & & \\
\hline
\end{tabular}

¿Cuál es el espacio muestral del experimento?

17. La probabilidad de un evento es igual al cociente de las frecuencias absolutas simples entre el total de las frecuencias. Calculen las siguientes probabilidades:
$P($ Todos ponen $)=$
$P($ Toma uno $)=$
$P($ Toma dos $)=$
$P($ Toma todo $)=$
$P($ Pon uno $)=$
$P($ Pon dos $)=$

18. ¿Todas las probabilidades son iguales?

19. Cuando $N$ (número de giros crece), ¿la probabilidad tiende a estabilizarse hacia un número en particular? ¿Cuál?

20. Comparen este número con el de sus compañeros. ¿Se parecen?

21. Anoten en la siguiente tabla los resultados que obtuvieron sus compañeros para el caso de 125 giros.

\begin{tabular}{|c|c|c|c|c|c|c|c|}
\hline & & $P$ (Todos ponen) $\mid$ & $P($ Toma uno $)$ & $P$ (Toma dos) & $P($ Toma todo $)$ & $P($ Pon uno $)$ & $P$ (Pon dos) \\
\hline \multirow{11}{*}{$\frac{\circ}{\frac{0}{3}}$} & A & & & & & & \\
\hline & B & & & & & & \\
\hline & C & & & & & & \\
\hline & D & & & & & & \\
\hline & E & & & & & & \\
\hline & $\mathrm{F}$ & & & & & & \\
\hline & G & & & & & & \\
\hline & $\mathrm{H}$ & & & & & & \\
\hline & 1 & & & & & & \\
\hline & J & & & & & & \\
\hline & K & & & & & & \\
\hline \multicolumn{8}{|c|}{ Suma } \\
\hline & edio & & & & & & \\
\hline
\end{tabular}

22. Sumen las probabilidades de cada columna y obtengan su promedio.

23. ¿Todas las probabilidades son iguales?

24. ¿La probabilidad $P(i)$ tiende a estabilizarse hacia un número en particular? ¿Cuál? 